\title{
Incidental Findings on Myocardial Perfusion SPECT Images
}

\author{
Razi Muzaffar ${ }^{1}$, Osama Raslan ${ }^{1}$, Fatma Ahmed ${ }^{1}$, Leonard Goldfarb ${ }^{2}$, Barbara Sterkel ${ }^{2}$, and Medhat M. Osman ${ }^{1,2}$ \\ ${ }^{1}$ Division of Nuclear Medicine, Department of Radiology, Saint Louis University, Saint Louis, Missouri; and ${ }^{2}$ Division of Nuclear \\ Medicine, Department of Radiology, John Cochran VA Medical Center, St. Louis, Missouri
}

CE Credit: For CE credit, you can access the test for this article, as well as additional JNMT CE tests, online at https://www.snmmilearningcenter.org. Complete the test online no later than September 2020. Your online test will be scored immediately. You may make 3 attempts to pass the test and must answer $80 \%$ of the questions correctly to receive $1.0 \mathrm{CEH}$ (continuing education hour) credit. SNMMI members will have their CEH credit added to their VOICE transcript automatically; nonmembers will be able to print out a CE certificate upon successfully completing the test. The online test is free to SNMMI members; nonmembers must pay $\$ 15.00$ by credit card when logging onto the website to take the test.

Myocardial perfusion SPECT plays a crucial role in the diagnosis of coronary artery disease, providing a noninvasive tool to monitor ischemia and infarction. The findings can have a profound impact on diagnosis and management in these patients. However, incidental noncardiac findings on myocardial perfusion SPECT images can also affect management. These noncardiac subtleties are most often detected on the rotating raw SPECT images, which are often overlooked. The intent of this pictorial essay is to illustrate several subtle noncardiac abnormalities within nuclear cardiac imaging that can have an impact on patient management and follow-up.

Key Words: nuclear cardiology; SPECT; 99mTc-sestamibi; myocardial perfusion SPECT

J Nucl Med Technol 2017; 45:175-180

DOI: 10.2967/jnmt.117.195487

\section{$\mathbf{T}$} he overall leading cause of death in the United States continues to be coronary artery disease, with costs in excess of $\$ 400$ billion spent each year on cardiovascular disease alone (1). As the life expectancy of the population continues to increase, the risk that heart disease will occur at some point in a person's lifetime increases as well. Myocardial perfusion imaging using SPECT continues to be an important noninvasive tool for clinicians to evaluate suspected or known coronary artery disease (2). However, in addition to the cardiac findings, incidental noncardiac findings can also be seen on the rotating raw SPECT images or on the CT images acquired for attenuation correction (3). These findings may lead to early diagnosis of a noncardiac condition that requires further treatment (4). Therefore, both cardiac and noncardiac findings need to be carefully evaluated.

Received May 2, 2017; revision accepted May 26, 2017.

For correspondence or reprints contact: Medhat M. Osman, Division of Nuclear Medicine, Department of Radiology, Saint Louis University, 3635 Vista Ave., 2-DT, St. Louis, MO 63110.

E-mail: mosman@slu.edu

Published online Jul. 13, 2017

COPYRIGHT @ 2017 by the Society of Nuclear Medicine and Molecular Imaging.
This pictorial essay presents various noncardiac findings on myocardial perfusion SPECT/CT images. We logged cases of incidental noncardiac abnormalities, reviewed the myocardial perfusion SPECT/CT images (along with supporting images from other modalities when available), and selected those that were most interesting.

\section{FINDINGS ON ROTATING RAW SPECT IMAGES}

\section{Lung Findings}

The primary use of myocardial perfusion SPECT is for the evaluation of myocardial perfusion; however, significant information can be attained from the rotating raw SPECT images of both the heart and the surrounding structures. One example is a small pericardial effusion, which can be visualized as subtle changes surrounding the heart (Fig. 1). Another example is fetal dextrocardia, a rare condition (incidence $<1 \%$ ) with various congenital abnormalities, typically found incidentally (Fig. 2) (5).

Near the heart are the lungs, for which various abnormalities can be detected on the rotating raw SPECT images. One example is chronic obstructive pulmonary disease, a debilitating condition involved in nearly 3 million deaths in 2015 (6). Although subtle, chronic obstructive pulmonary disease is recognizable as a flattening of the diaphragm on the raw images (Fig. 3). Another example is pleural

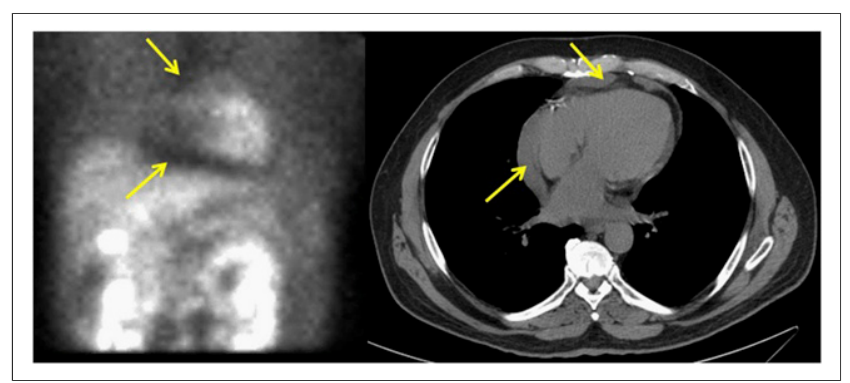

FIGURE 1. Patient who presented with chest pain and shortness of breath. Raw SPECT image shows small area of photopenia surrounding heart (arrows, left). CT image shows small pericardial effusion (arrows, right). 
FIGURE 2. Patient with situs inversus who presented with chest pain. Raw SPECT image (top left) shows enlarged spleen (S) in right abdomen, and liver in left abdomen (L). Processed images (top right) show lateral wall and septum flipped from normal position as evidenced by short septum; finding is most prominent in horizontal long axis (last 2 rows of processed images). CT images (bottom left and middle) and radiograph (bottom right) show that heart $(\mathrm{H})$ and major organs mirror their normal positions.

FIGURE 3. Patient with chronic obstructive pulmonary disease. Flattening of diaphragm is seen on raw SPECT stress (arrows, left) and rest (arrow, right) images, as well as on radiograph (arrows, center).
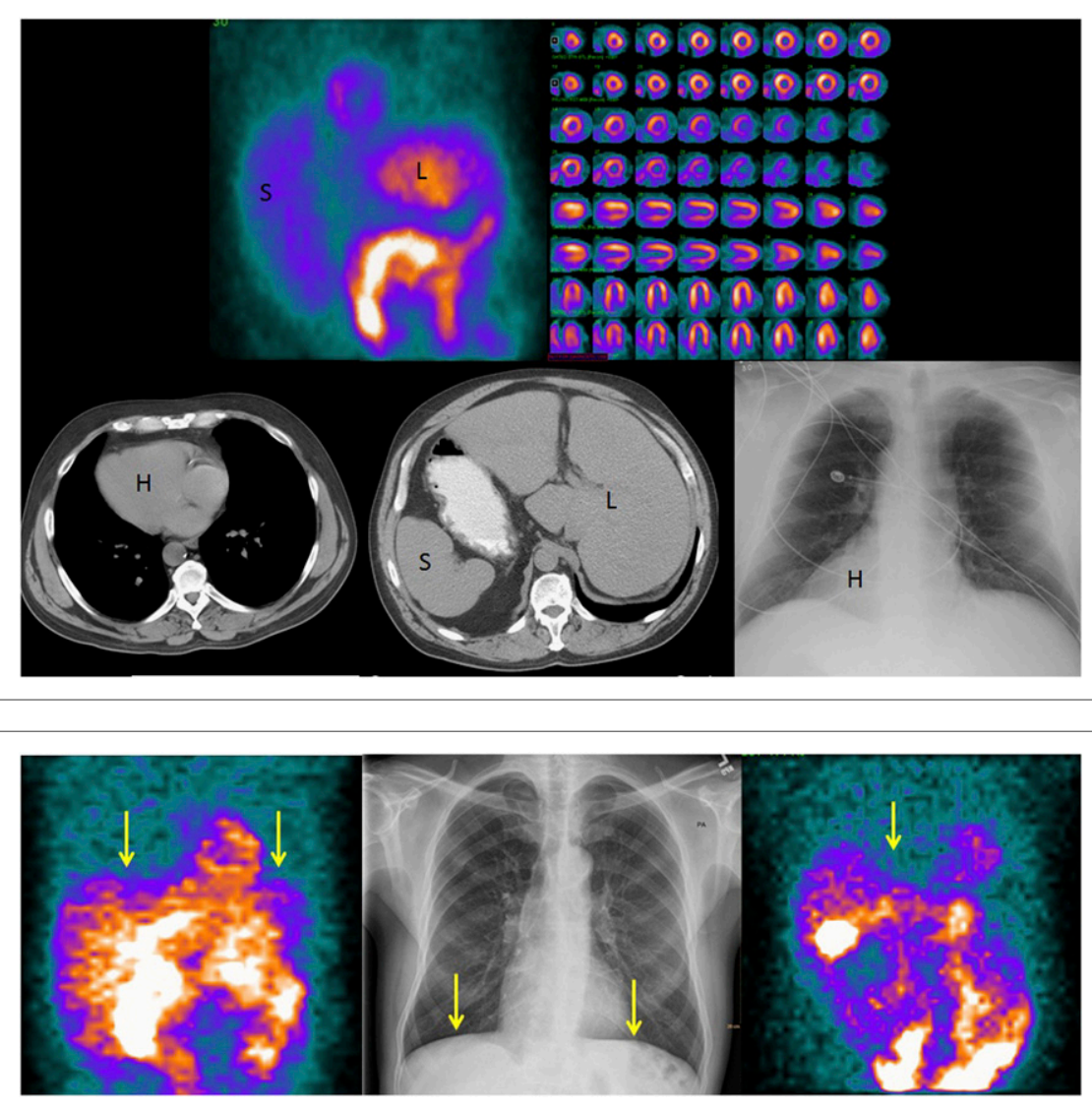

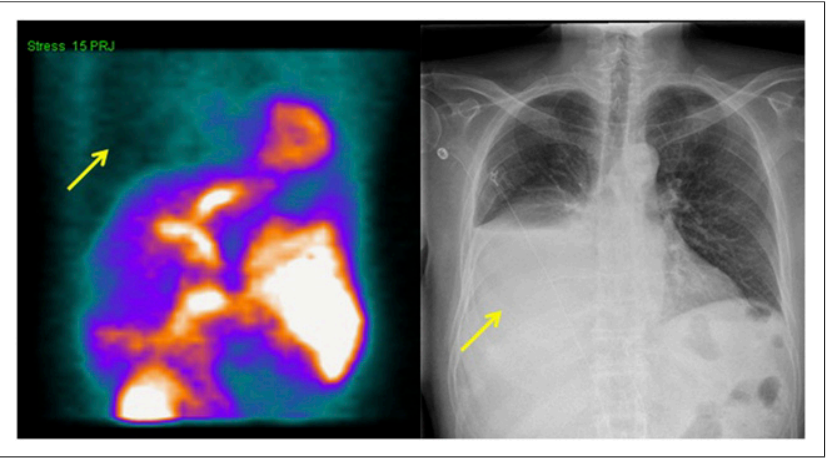

FIGURE 4. Patient with pleural effusion. Relative photopenia in right chest cavity on raw SPECT image (arrow, left) correlates with large pleural effusion on chest radiograph (arrow, right).

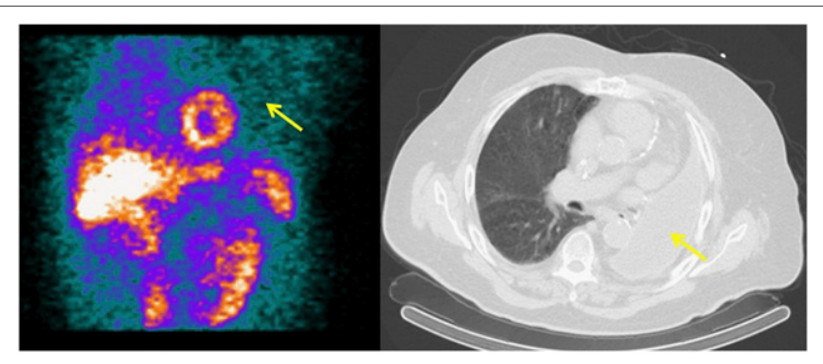

FIGURE 5. Lung cancer patient who was being evaluated after undergoing left pneumonectomy. Reduced counts in left chest on raw SPECT image (arrow, left) correlate with pneumonectomy changes on CT image (arrow, right). effusion, which often occurs in conjunction with a variety of disease states and can be seen as an area of reduced counts (Fig. 4). This pattern of photopenia can also be seen in patients who have undergone pneumonectomy (Fig. 5).

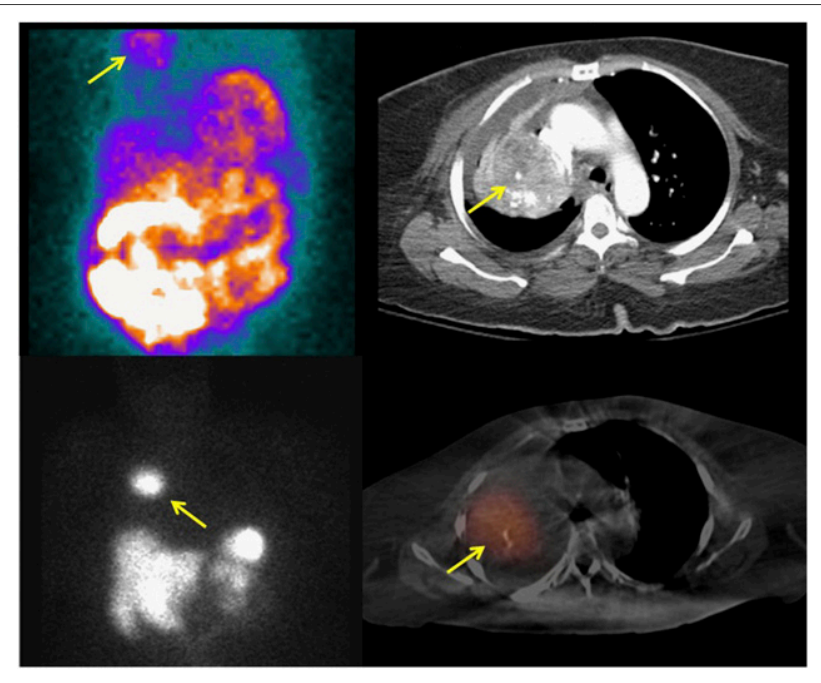

FIGURE 6. Patient with history of carcinoid tumor who presented with chest pain. Raw SPECT image shows mass in right upper chest (arrow, top left). Contrast-enhanced CT image shows heterogeneously enhancing mass in right lung (arrow, top right). ${ }^{111}$ In-pentetreotide SPECT image shows right-chest mass (arrow, bottom left), which fuses with right-lung mass on SPECT/CT image (arrow, bottom right). 


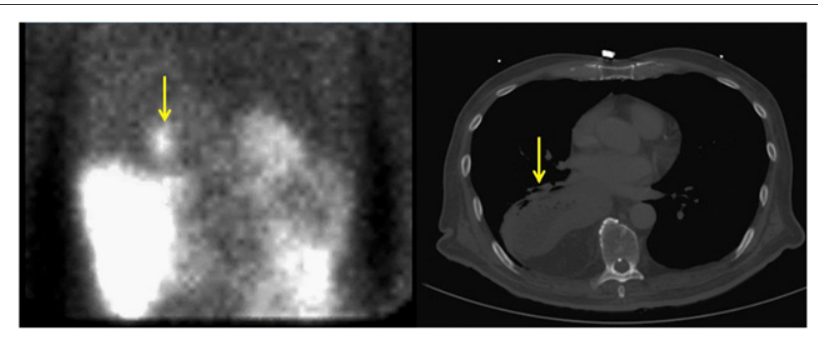

FIGURE 7. Patient who presented with chest pain. Raw SPECT image shows abnormal tracer accumulation in right chest (arrow, left). CT image demonstrates, in posterior right hemidiaphragm, large hernia containing nearly all of stomach (arrow, right).

One of the more common and well-known incidental noncardiac findings is lung cancer, which is the leading cause of cancer death worldwide among both men and women (7). A less common form of lung cancer that may be seen on the raw images is neuroendocrine tumors such as carcinoids-rare tumors that can occur within any tissue, including the lung (Fig. 6). Although pulmonary carcinoids are a common type of neuroendocrine tumor, they comprise only $1 \%-2 \%$ of all lung tumors (8).

Lung cancer can be mimicked on the raw images by gastric reflux with subsequent progression to hiatal hernia, for which patients may present with chest pain (Figs. 7-9). This finding exemplifies a common hurdle in the interpre-

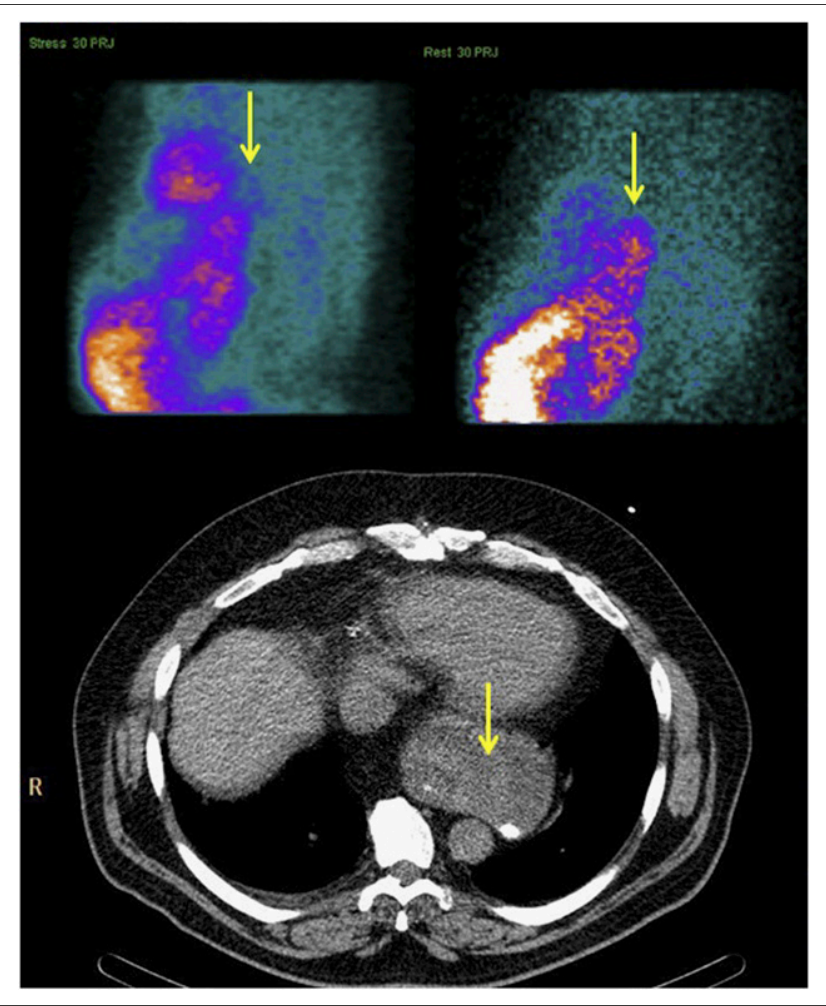

FIGURE 8. Patient with abnormal tracer accumulation posterior to heart on raw SPECT images, more pronounced at rest than at stress (arrows, top). CT image shows moderate hiatal hernia (arrow, bottom).

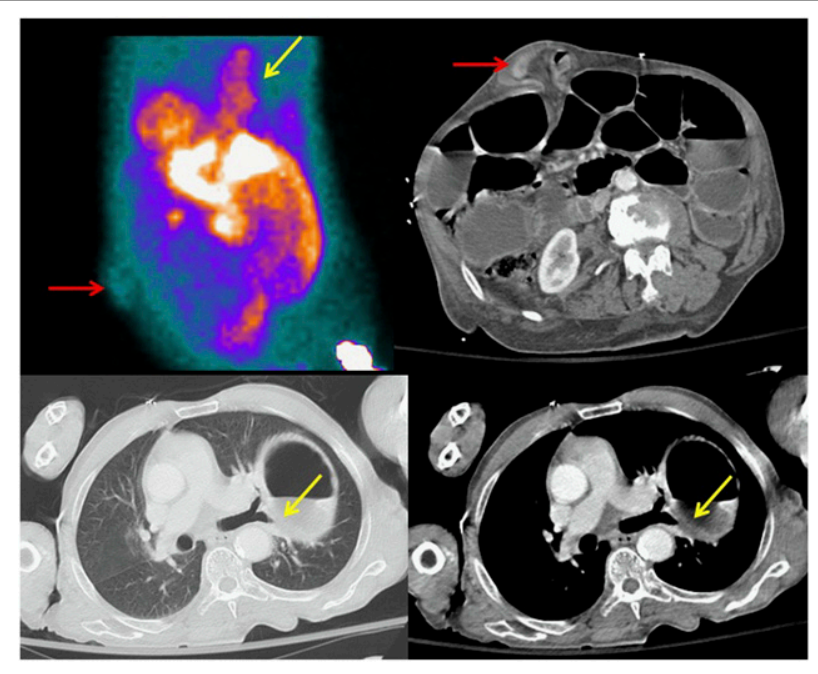

FIGURE 9. Patient who presented with chest pain. Raw SPECT image shows focus of uptake in anterior abdominal wall (red arrow, top left) and linear uptake in chest (yellow arrow, top left). CT images show ventral hernia (arrow, top right), as well as large hiatal hernia (yellow arrows, bottom).

tation of myocardial perfusion images - the presence of increased activity in the liver, bowel, or stomach, predominantly affecting the inferior wall-as all ${ }^{99 \mathrm{~m}} \mathrm{Tc}$-labeled myocardial perfusion agents are cleared by the liver and excreted by the biliary system into the duodenum (9). Various techniques have been used to reduce this uptake, with
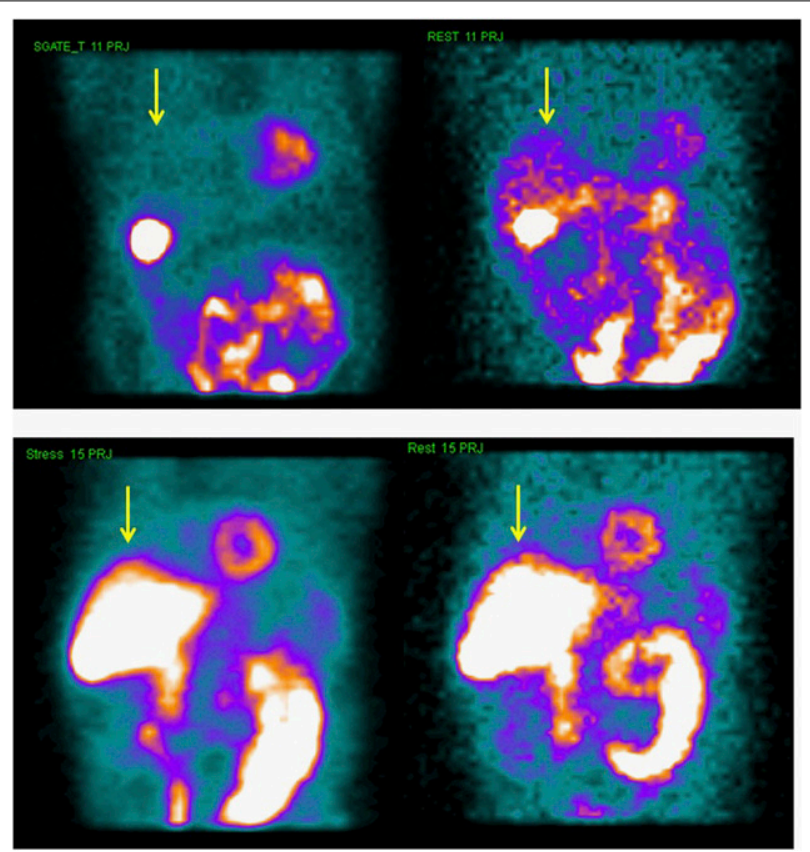

FIGURE 10. Patient who presented with chest pain. Raw SPECT images from treadmill stress test show minimal liver activity and greater distal bowel activity at stress (arrow, top left) than at rest (arrow, top right). Raw SPECT images from pharmacologic stress test show intense liver and bowel activity both at stress (arrow, bottom left) and at rest (arrow, bottom right). 
FIGURE 11. Patient who presented with chest pain. Raw SPECT image shows round area of increased uptake in right upper chest (arrow, left). Right-mediastinum mass is seen on axial CT image (arrow, center) and coronal CT image (arrow, right). On biopsy, mass was found to be thymoma.

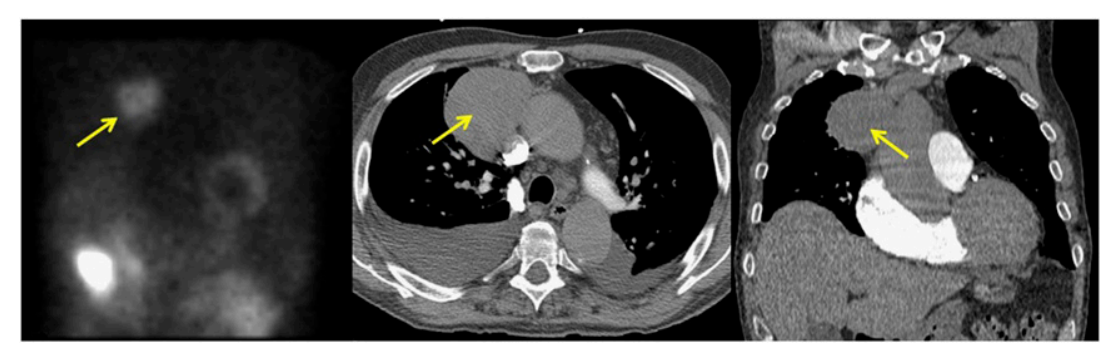

varying degrees of success (10). Although this drawback is usually encountered during the rest portion of the examination, it is becoming more common during the stress portion as well, because of the increased use of pharmacologic stress rather than treadmill stress (Fig. 10).

\section{Mediastinal Findings}

In addition to lung cancer, tumors of the mediastinum such as a thymoma can be seen on the rotating raw SPECT images (Fig. 11). Thymomas can be benign or malignant and account for nearly $20 \%$ of anterior mediastinal neoplasms in adults (11).

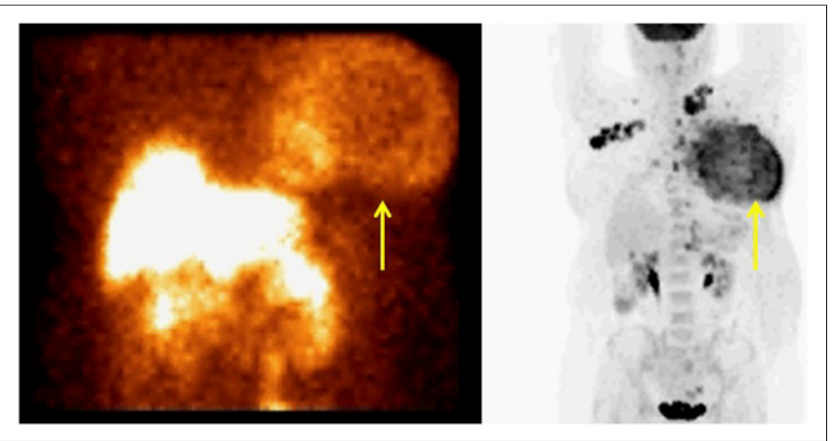

FIGURE 12. Female patient with recently diagnosed breast cancer who presented for preoperative risk assessment. Raw SPECT image shows large mass in left breast (arrow, left). Maximum-intensity-projection PET/CT image demonstrates large left-breast mass (arrow, right) with multiple small metastatic lymph nodes.

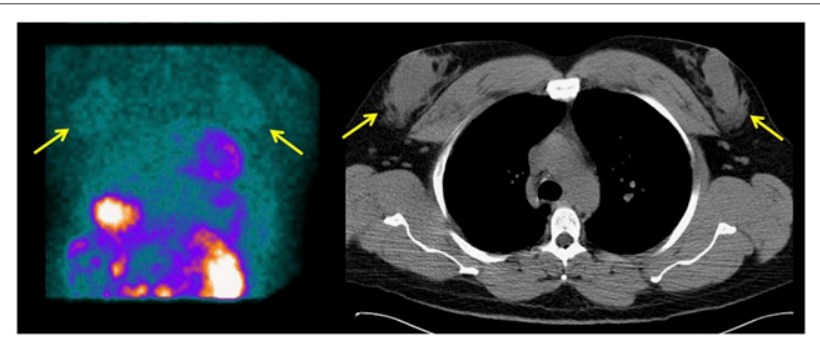

FIGURE 13. Male patient with end-stage liver disease who presented for pretransplantation evaluation. Raw SPECT image shows round areas of mildly increased uptake in chest (arrows, left) correlating with gynecomastia changes on CT image (arrows, right).

\section{Breast Findings}

Incidental areas of uptake on the rotating raw SPECT images have also been seen in the breast, usually because of

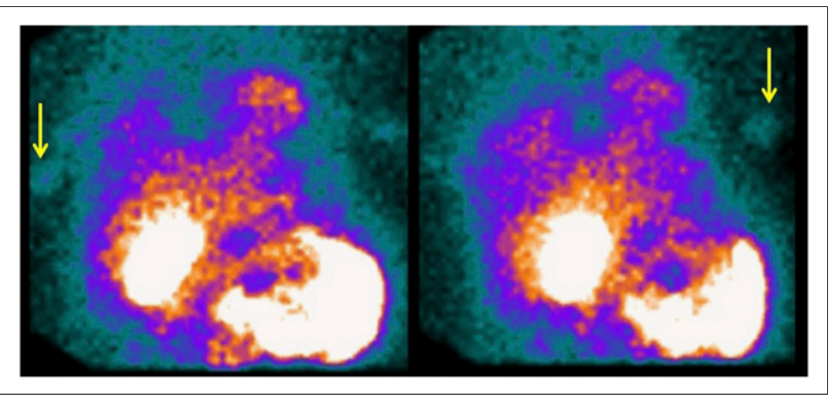

FIGURE 14. Female patient who presented with chest pain. Raw SPECT images show small areas of increased uptake bilaterally in chest (arrows). Clinical examination found abscesses bilaterally in lower breasts.

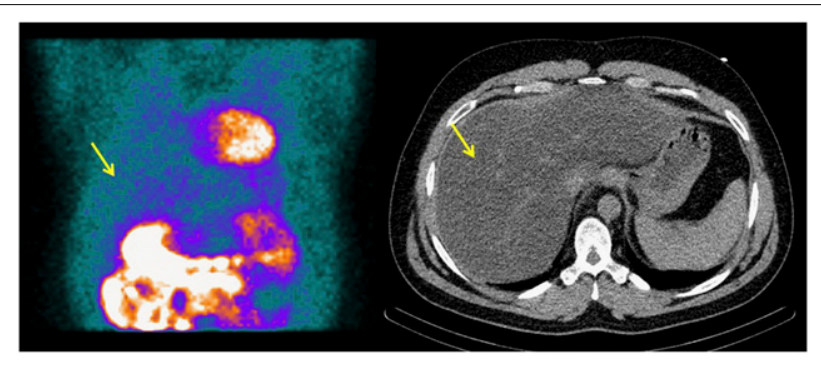

FIGURE 15. Patient with fatty liver disease. Raw SPECT image shows minimal uptake in liver (arrow, left). CT image shows diffuse fatty changes throughout liver (arrow, right).

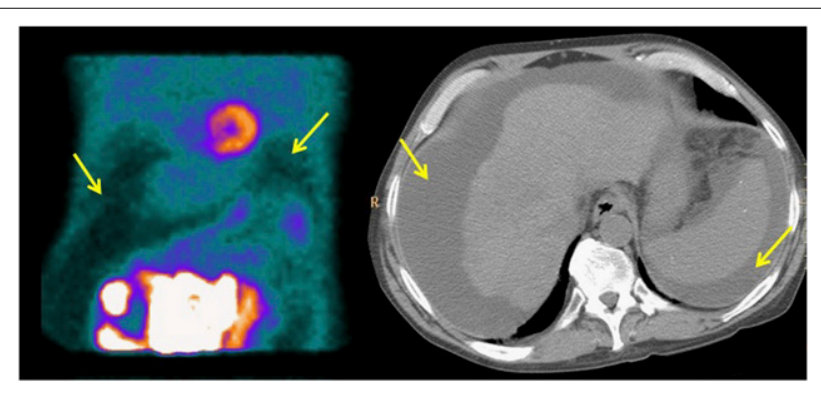

FIGURE 16. Patient with end-stage liver disease who presented for pretransplantation evaluation. Raw SPECT image shows areas of photopenia throughout abdomen (arrows, left). CT image shows diffuse ascites surrounding liver and spleen (arrows, right). 


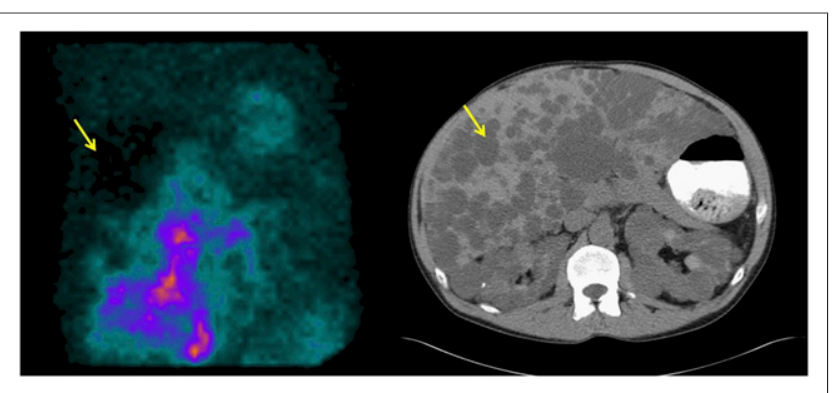

FIGURE 17. Patient with end-stage renal disease who presented for preoperative evaluation. Raw SPECT image demonstrates relative photopenia in liver (arrow, left). CT image shows polycystic hepatic disease (arrow, right) and renal disease.

malignancy (Fig. 12) (12,13). However, nonmalignant causes of radiotracer accumulation in the breast have also been seen. For example, gynecomastia in men - a benign proliferation of glandular tissue in the breast that is often a precursor of an underlying disease-is a common finding for various reasons (Fig. 13) (14,15). Furthermore, areas of infection within the breast may show focal radiotracer accumulation (Fig. 14).

\section{Liver Findings}

Several types of liver abnormality can alter the radiotracer distribution seen on the rotating raw SPECT images.
One example is nonalcoholic fatty liver disease, which affects $25 \%-30 \%$ of the population and can lead to cardiovascular diseases, cirrhosis, or hepatocellular cancer (16). The effects of fatty liver can be seen as an intrahepatic area of photopenia (Fig. 15). Another example is ascites, a common complication of cirrhosis (Fig. 16). If liver disease progresses to liver failure requiring liver transplantation, the heart must be evaluated before the surgery, as the prevalence of coronary artery disease in patients requiring liver transplantation ranges from $7.1 \%$ to $28 \%$. As part of the cardiac work-up, myocardial perfusion imaging is often used in conjunction with other screening measures (17).

\section{Other Abdominal Findings}

The literature has described other causes of abdominal photopenia on the rotating raw SPECT images, such as simple hepatic cysts (Fig. 17), echinococcal cysts, cholecystitis (18), and tumors (Fig. 18).

\section{FINDINGS ON ATTENUATION-CORRECTION CT IMAGES}

With the advancement of technology and the increased availability of SPECT/CT, many facilities perform myocardial perfusion SPECT with CT attenuation correction
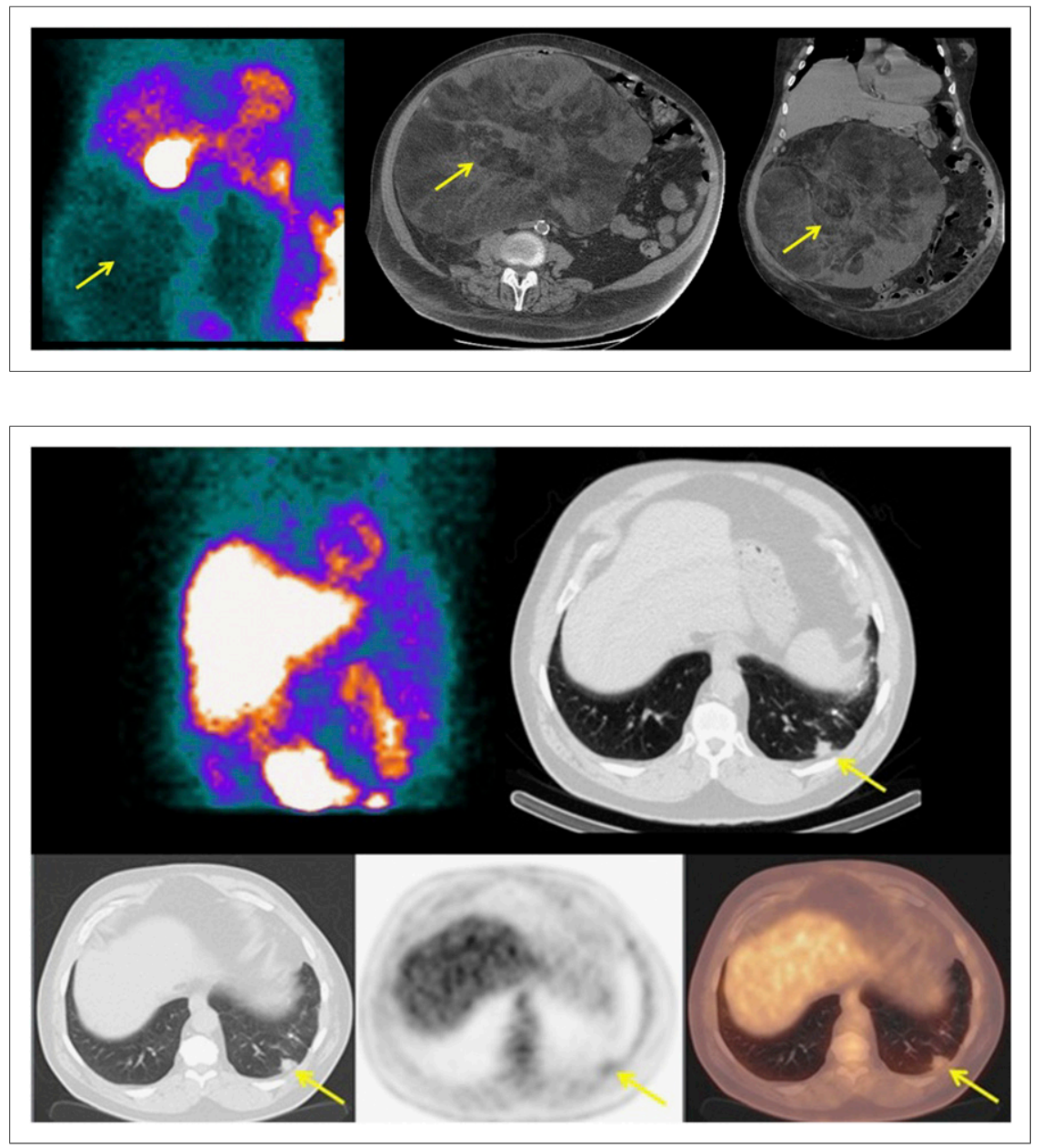

FIGURE 18. Patient with large abdominal mass who presented for preoperative evaluation. Raw SPECT image shows large area of photopenia within abdomen (arrow, left). CT images show large intraabdominal fatty mass (arrows, middle and right), which biopsy found to be welldifferentiated liposarcoma.
FIGURE 19. Patient who presented with chest pain. Raw SPECT image is unremarkable (top left). CT image obtained for attenuation correction shows subcentimeter spiculated nodule in left lung base (arrow, top right). PET/CT image shows mild ${ }^{18} \mathrm{~F}$ FDG uptake in nodule (arrows, bottom row), with SUV max of 2.5. Biopsy was performed and found adenocarcinoma. 
FIGURE 20. Patient with history of achalasia who presented with chest pain. Raw SPECT image is unremarkable (left). CT images obtained for attenuation correction show significant esophageal dilation (arrows, middle and right), consistent with patient history.

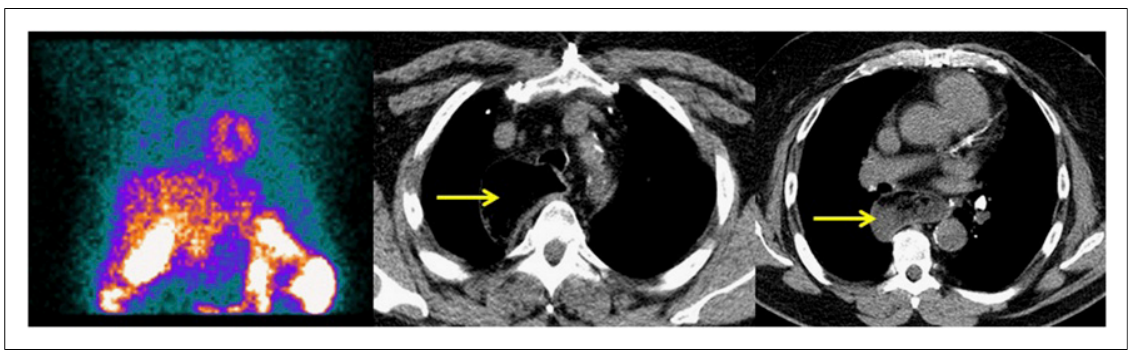

FIGURE 21. Various subtle abnormalities on CT images obtained for attenuation correction: small hemangioma in body of T12 (yellow arrow), subcentimeter exophytic isodensity in right kidney (red arrow), and small adenoma in left adrenal gland (green arrow).

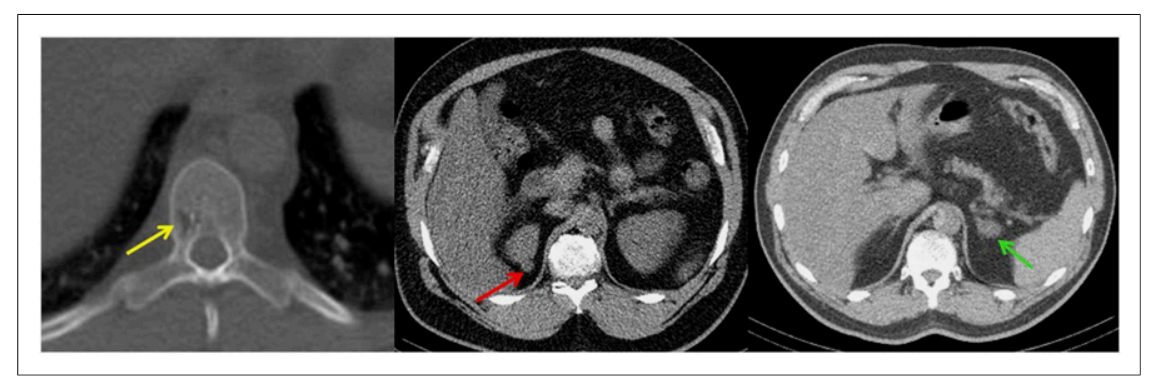

to improve diagnostic accuracy. This additional layer of imaging allows for the detection of innumerable findings, both benign and malignant, not apparent on the myocardial perfusion SPECT images themselves (Figs. 19-21) (3). A recent study demonstrated that nearly $70 \%$ of all myocardial perfusion SPECT/CT examinations have both minor and major extracardiac findings on the attenuationcorrection CT images (19). Of the major extracardiac findings in that study, more than half $(52.6 \%)$ were previously unknown. The study emphasized the value of reviewing the $\mathrm{CT}$ portion of the examination and shed light on the amount of information that can be obtained from one of the most commonly performed nuclear medicine examinations.

\section{CONCLUSION}

Myocardial perfusion imaging with SPECT or SPECT/ CT is a valuable tool for evaluating suspected or known coronary artery disease but can also detect and diagnose a variety of other diseases. Regardless of whether the findings are apparent or subtle, they can have a profound impact on patient management and follow-up. The detection and reporting of these subtle abnormalities often require careful inspection of both the rotating raw SPECT images and the CT images obtained for attenuation correction. Therefore, interpretation of myocardial perfusion images should not be limited to merely evaluation of the heart.

\section{REFERENCES}

1. Hendrani AD, Adesiyun T, Quispe R, et al. Dyslipidemia management in primary prevention of cardiovascular disease: current guidelines and strategies. World J Cardiol. 2016;8:201-210.

2. Gimelli A, Liga R, Duce V, et al. Accuracy of myocardial perfusion imaging in detecting multivessel coronary artery disease: a cardiac CZT study. J Nucl Cardiol. 2017;24:689-695.
3. Coward J, Lawson R, Kane T, et al. Multi-centre analysis of incidental findings on low-resolution CT attenuation correction images. Br J Radiol. 2014;87:20130701.

4. Chatziioannou SN, Alfaro-Franco C, Moore WH, et al. The significance of incidental noncardiac findings in Tc-99m sestamibi myocardial perfusion imaging: illustrated by a case. Tex Heart Inst J. 1999;26:229-231.

5. Bernasconi A, Azancot A, Simpson JM, et al. Fetal dextrocardia: diagnosis and outcome in two tertiary centres. Heart. 2005;91:1590-1594.

6. Chronic obstructive pulmonary disease (COPD): fact sheet. World Health Organization website. http://www.who.int/mediacentre/factsheets/fs315/en/. Published November 2016. Accessed June 28, 2017.

7. Torre LA, Siegel RL, Jemal A. Lung cancer statistics. Adv Exp Med Biol. 2016;893:1-19.

8. Hilal T. Current understanding and approach to well differentiated lung neuroendocrine tumors: an update on classification and management. Ther Adv Med Oncol. 2017;9:189-199.

9. Erdoğan Z, Silov G, Ozdal A, et al. Enterogastroesophageal reflux detected on $99 \mathrm{~m}$-technetium sestamibi cardiac imaging as a cause of chest pain. Indian $J$ Nucl Med. 2013;28:45-48.

10. van Dongen AJ, van Rijk PP. Minimizing liver, bowel, and gastric activity in myocardial perfusion SPECT. J Nucl Med. 2000;41:1315-1317.

11. Benveniste MF, Moran CA, Mawlawi O. FDG PET-CT aids in the preoperative assessment of patients with newly diagnosed thymic epithelial malignancies. J Thorac Oncol. 2013;8:502-510.

12. Ammar KA, Shaikh A, Anigbogu M, et al. Breast cancer diagnosed by stress SPECT sestamibi: the role of inverse gray-scale imaging. J Nucl Cardiol. September 1, 2016 [Epub ahead of print].

13. García-Talavera P, Olmos R, Sainz-Esteban A, et al. Evaluation by SPECT-CT of an incidental finding of a thymoma and breast cancer in a myocardial perfusion SPECT with ${ }^{99 m}$ Tc-MIBI. Rev Esp Med Nucl Imagen Mol. 2013;32: $260-262$.

14. Uthamalingam S, Sidhu MS, Gurm GS, et al. Male breast uptake of $99 \mathrm{~m}-\mathrm{Tc}$ sestamibi in myocardial perfusion imaging. Int J Cardiol. 2012;155:e22e23.

15. Mieritz MG, Christiansen P, Jensen MB, et al. Gynaecomastia in 786 adult men: clinical and biochemical findings. Eur J Endocrinol. 2017;176:555-566.

16. Bellentani S. The epidemiology of non-alcoholic fatty liver disease. Liver Int. 2017;37(suppl 1):81-84

17. Ye C, Saincher M, Tandon P, et al. Cardiac work-up protocol for liver transplant candidates: experience from a single liver transplant centre. Can J Gastroenterol. 2012;26:806-810.

18. Lyon J, Spaulding J, Zack PM. Large abdominal photopenic area on ${ }^{99 \mathrm{~m}} \mathrm{Tc}$ sestamibi myocardial perfusion imaging. J Nucl Med Technol. 2012;40:281-282.

19. Zadro C, Roussel N, Cassol E, et al. Prognostic impact of myocardial perfusion single photon emission computed tomography in patients with major extracardiac findings by computed tomography for attenuation correction. $\mathrm{J} \mathrm{Nucl} \mathrm{Car-}$ diol. March 9, 2017 [Epub ahead of print]. 